\title{
Modeling Slip System Strength Evolution in Ti-7Al Informed by In-Situ Grain Stress Measurements
}

\author{
Darren C. Pagan ${ }^{1}$, Paul A. Shade ${ }^{2}$, Nathan R. Barton ${ }^{1}$, Jun-Sang Park ${ }^{3}$, Peter Kenesei \\ ${ }^{3}$, David B. Menasche ${ }^{4}$, Joel V. Bernier ${ }^{1}$ \\ ${ }^{1}$ Lawrence Livermore National Laboratory \\ ${ }^{2}$ Air Force Research Laboratory \\ ${ }^{3}$ Advanced Photon Source, Argonne National Laboratory \\ ${ }^{4}$ Carnegie Mellon University
}

\begin{abstract}
Far-field high-energy X-ray diffraction microscopy is used to asses the evolution of slip system strengths in hexagonal close-packed (HCP) Ti-7Al during tensile deformation in-situ. The following HCP slip system families are considered: basal $\langle a\rangle$, prismatic $\langle a\rangle$, pyramidal $\langle a\rangle$, and first-order pyramidal $\langle c+a\rangle$. A $1 \mathrm{~mm}$ length of the specimen's gauge section, marked with fiducials and comprised of an aggregate of over 500 grains, is tracked during continuous deformation. The response of each slip system family is quantified using 'slip system strength curves' that are calculated from the average stress tensors of each grain over the applied deformation history. These curves, which plot the average resolved shear stress for each slip system family versus macroscopic strain, represent a mesoscopic characterization of the aggregate response. A short time-scale transient softening is observed in the basal $\langle a\rangle$, prismatic $\langle a\rangle$, and pyramidal $\langle a\rangle$ slip systems, while a long time-scale transient hardening is observed in the pyramidal $\langle c+a\rangle$ slip systems. These results are used to develop a slip system strength model as part of an elasto-viscoplastic constitutive model for the single crystal behavior. A suite of finite element simulations is performed on a virtual polycrystal to demonstrate the relative effects of the different parameters in the slip system strength model. The model is shown to accurately capture the macroscopic stress-strain response using parameters that are chosen to capture the mesoscopic slip system responses.
\end{abstract}

Preprint submitted to Acta Materialia

January 16, 2017

(C) 2017. This manuscript version is made available under the Elsevier user license http://www.elsevier.com/open-access/userlicense/1.0/ 


\section{Introduction}

The adoption of an integrated computational materials engineering (ICME) approach for the design of critical polycrystalline structural components requires the development of rigorously validated micromechanical models. These models must acurately predict elasto-

5 plastic response in-operando across multiple length scales [1,2]. An important length scale for understanding and characterizing critical phenomena, such as incipient damage nucleation and growth, is that which comprises a local neighborhood of grains. Heterogeneities in the local intergranular stresses and strains arise even under simple macroscopic loading conditions due to both the anisotropic elastic and plastic properties of the constituent grains

10 (particularly for hexagonal metals) as well as complex interactions among them. These heterogeneities can vary significantly from the mean fields and give rise to 'hot spots' that have a higher probability of nucleating and propagating voids or cracks [3, 4]. Therefore, a mesoscopic characterization of both the individual crystal responses as well as the aggregate interactions is critical to the formulation and validation of accurate micromechanical models. The measured volume at a given macroscopic load must therefore span an aggregate large enough to sample the full range of grain loading states. Far-field high-energy X-ray diffraction microscopy (ff-HEDM) provides such a mesoscopic characterization of evolving microstructure and micromechanical state for polycrystalline specimens subject to thermomechanical processing in-situ [5]. We demonstrate this by using lattice strain data measured

20 by ff-HEDM to directly develop and calibrate a single crystal constitutive model capable of also capturing the aggregate response; specifically a per-mechanism slip system strength model for Ti-7Al.

Using the ff-HEDM technique at third generation synchrotron sources, the stress states in aggregates of up to $\approx 1000$ grains have been studied during in-situ deformation. These data, taken as an ensemble, are a boon to the advancement of predictive models for the mechanical response of polycrystalline materials $[6,7]$. Relevant to this work, the technique has been applied to the measurement of stresses in individual grains of titanium alloys during in-situ deformation $[8,9,10,11]$. In practice however, extracting relevant quantities from these large, high-dimensional data sets present an open challenge; new methods must be developed to distill down these massive quantities of data so that they can be used to enhance our physical understanding of deformation processes, and in turn inform micromechanical models. Towards this goal, we focus on a method to extract the per-family evolution of slip 
system strengths in Ti-7Al using ff-HEDM and use these data to formulate and calibrate a micromechanical model that accurately captures the meso- and macroscale flow responses.

Crystal plasticity formulations are based on the principle of restricted slip. A family of slip systems is comprised of all symmetrically equivalent combinations of slip plane and direction that can accommodate shear deformation. Strength, in rate-dependent viscoplastic formulations, is a state based quantity relating the resolved shear stress to shear rate. Hardening is represented with the evolution equations for the strength quantities. Accu40 rate values for initial slip system strength are necessary for proper representation of the development of incipient plastic flow. Notably, the onset of yield is a critical portion of the mechanical response during both fatigue and fracture processes in ductile crystalline materials. Modeling the evolution of slip system strengths also requires accurate modeling parameters. However, while slip system strength and its evolution are important compo-

45 nents of nearly all microscale plasticity models, most modeling efforts settle for calibrations that are based on indirect quantities such as flow strength and texture evolution.

The Ti-7Al tested herein exists in the hexagonal close-packed (HCP) $\alpha$ phase at room temperature and is similar in composition to the $\alpha$ phase of several commercial alloys, including Ti-6Al-4V. Ti-7Al serves as a model for materials that exhibit both significant non-uniformities among slip system strengths and evolution of the slip system strengths with increasing strain. Plastic deformation in Ti-7Al is accommodated primarily by slip on the basal $\langle a\rangle(\langle 11 \overline{2} 0\rangle(0001))$ and prismatic $\langle a\rangle(\langle 11 \overline{2} 0\rangle\{1 \overline{1} 00\})$ slip systems and less frequently, slip is also observed on the pyramidal $\langle a\rangle(\langle 11 \overline{2} 0\rangle\{1 \overline{1} 01\})$ slip systems [12]. The final deformation mode necessary to close the single crystal yield surface is provided by 55 the glide of $\langle c+a\rangle$ dislocations on $\{10 \overline{1} 1\}$ and $\{11 \overline{2} 2\}$ families of planes (first order and second order pyramidal slip respectively) $[13,14,15]$, rather than by twinning as in pure titanium. With the inclusion of these slip systems, the von Mises criterion is satisfied and all modes of plastic deformation can be accommodated. Previous work has shown that various families of slip systems in titanium exhibit significant variation in initial slip system strengths, producing significant anisotropy of the shape of the single crystal flow surface [12].

In addition to variation of initial slip system strengths, aging of Ti-7Al leads to shortrange ordering (SRO) and the formation of nanoscale coherent $\alpha_{2}$ precipitates $\left(\mathrm{Ti}_{3} \mathrm{Al}\right)$ [16]. The existence of SRO and these $\alpha_{2}$ precipitates is known to influence the mechanical response 
65 of titanium alloys with aluminum content greater than 5\% [12]. First, the presence of the $\alpha_{2}$ precipitates has been reported to supress twinning [17]. Also, slip is enhanced on the basal plane [18], promoting intense slip localization and banding [19, 20, 15]. Lastly, the $\mathrm{SRO}$ and $\alpha_{2}$ precipitates can serve as dislocation obstacles that raise the strength of the material [12], but as slip occurs these obstacles are sheared by dislocation motion and lose their effectiveness as slip barriers [19], effectively softening the material with increasing macroscopic strain [21].

Measuring the intial slip system strengths of a material such as Ti-7Al is a difficult process, and quantifying the hardening or softening behavior of a slip system even more so. If possible, a single crystal is grown and oriented such that uniaxial deformation will be resolved shear stress applied to the assumed active slip system when elastic behavior ends [15]. In these single crystal tests, the evolution of the strength becomes more difficult to quantify because other slip systems activate and begin to interact with one another with increasing load, particularly in HCP metals. Furthermore, the single crystal experiments

so are typically conducted under uniaxial stress loading conditions, whereas grains inside of a deforming polycrystal are subject to a wide array of local deformation conditions that may influence the hardening response. In lieu of calibration to macroscopic quantities, we propose a method that uses the individual stress responses of an ensemble of grains (single crystals) in a deforming aggregate to constrain the model. Each grain probes different portions of the single crystal flow surface; as an ensemble, they can be used to determine the flow surface's shape and evolution.

In this paper, we isolate the effects of individual slip mechanisms by extracting the evolution of slip system strengths from the ensemble data gathered from an aggregate of individually resolved grains via ff-HEDM. Virtual samples are generated using a simple Voronoi tessellation of measured grain centroids and lattice orientations, facilitating direct comparisons between resolved shear stress distributions from both the simulated and experimental results. The slip system strength data are then used to test a single crystal strength evolution model for Ti-7Al as part of an elasto-viscoplastic constitutive model. The effects of different components of the slip system strength model are explored by modulating 95 individual parameters in polycrystal plasticity simulations. 


\section{Experiment}

\subsection{Material}

The material studied in this work is the titanium alloy Ti-7Al (nominal composition Ti-7.02Al-0.11O-0.015Fe wt\%). The material was cast as an ingot, hot isostatic pressed, extruded, annealed at $962^{\circ} \mathrm{C}$ for $24 \mathrm{~h}$, and then air cooled [22]. The processing route produced relatively large, equiaxed grains $(75 \mu \mathrm{m}$ diameter $)$ with relatively little intra-granular misorientation, as was evidenced by sharp diffraction peaks observed in the undeformed state. Titanium alloys, including the one studied in this work, have high strength to stiffness ratios that allow the lattice to undergo large elastic deformations prior to the onset of plastic deformation.

\subsection{Experiment Description}

A Ti-7Al sample was deformed in uniaxial tension at the 1-ID-E beamline at the Advanced Photon Source. The sample was deformed within the RAMS1 load frame insert [23] contained in a servohydraulic MTS load frame. The cross sectional area of the sample was $1 \mathrm{~mm} \times 1 \mathrm{~mm}$ and the gauge section was $8 \mathrm{~mm}$ in length. The sample was loaded with a constant crosshead velocity of $1.6 \mathrm{~nm} / \mathrm{s}$. Macroscopic strains were calculated from optical images of the electrical discharge machined specimen surface using two point digital image correlation (DIC) of surface feature displacements. The DIC analysis was conducted using a Matlab script and a discrete fourier transform based subpixel image registration algorithm [24]. From the DIC analysis performed on the specimen surface, strain rates in the diffraction volume varied from $10^{-7} \mathrm{~s}^{-1}$ to $10^{-6} \mathrm{~s}^{-1}$ during testing. Macroscopic stress was calculated using output from a load cell placed at the top of the sample. A pair of gold fiducial markers were adhered to the sample surface and utilized as a reference throughout the experiment to ensure that the same volume of material was interrogated by the X-ray through out the ff-HEDM measurements. [25].

The macroscopic stress-strain curve is shown in Figure 1. The circles correspond to the beginning of intervals where diffraction and tomographic data were collected. The sample macroscopically yielded at $530 \mathrm{MPa}$ with a distinct yield point. After yielding, there was a small drop in the flow stress to $525 \mathrm{MPa}$ followed by work hardening to $535 \mathrm{MPa}$ by the end of the test. Overall, the evolution of the macroscopic flow stress was minimal during the test. 
At multiple time points as the sample was deformed, two complete $360^{\circ}$ rotations (sweeps) about the loading axis were performed. One sweep was performed to collect radiographs for tomographic reconstructions, measured in case of the event of a void or crack nucleating; and a second sweep was performed to collect diffraction peaks from many sets of lattice planes as required by the ff-HEDM technique. The images in each rotation sweep were collected in $0.25^{\circ}$ intervals for a total of 1440 images per sweep and the combined collection time for the tomography and diffraction sweeps was approximately 42 minutes. In a typical quasi-static in-situ experiment, loading is halted and the material is removed from the flow surface to make ff-HEDM measurements $[8,26]$. However for this experiment, the slow crosshead velocity was chosen such that material and stress evolution were negligible during the time it took to complete a rotation sweep, allowing data to be collected without halting loading. Some implications of the chosen loading rate will be discussed.

A diagram of the experiment is given in Figure 2. The subscript L indicates the laboratory coordinate system, $\mathrm{S}$, the sample coordinate system, and $\mathrm{D}$, the detector coordinate system. A full $1 \mathrm{~mm}$ tall cross section of the sample was illuminated with a $61.332 \mathrm{keV}$ $\mathrm{X}$-ray beam (tuned to the ytterbium $\mathrm{K}$ absorption edge) that traveled in the $-\boldsymbol{e}_{\boldsymbol{z}}^{L}$ direction. Diffracted intensity was collected on a large panel area detector that was located $756 \mathrm{~mm}$ downstream of the sample. The detector was a GE amorphous silicon area detector with $2048 \times 2048$ pixels and a $200 \mu \mathrm{m}$ pixel size [27]. The orientation of the detector is described by the detector coordinate system where $\boldsymbol{e}_{\boldsymbol{x}}^{D}$ and $\boldsymbol{e}_{\boldsymbol{y}}^{D}$ are aligned with the edges of the detector and $\boldsymbol{e}_{\boldsymbol{z}}^{D}$ is normal to the detector face. The instrument geometry calibration procedure is found in [28]. For rotation sweeps, the sample was rotated about the $\boldsymbol{e}_{\boldsymbol{y}}^{L}$ direction by the angle $\omega$. The sample and laboratory coordinate systems are aligned when $\omega=0^{\circ}$.

In total, diffraction and tomography scans were taken at 58 points along the stress strain curve as the gauge section of the sample was deformed to a macroscopic strain of 0.025. Using the ff-HEDM data, 507 individual grains were successfully indexed and the centroids, lattice orientations, and lattice strain tensors from these grains were found at the 58 measurement points.

\subsection{Diffraction Data Processing}

Measured positions from multiple diffraction peaks were used to determine each grain's centroid (3 parameters), lattice orientation ( 3 parameters), and elastic strain state (6 parameters) using the HEXRD software package (https://github.com/joelvbernier/hexrd). A 
sufficient number of independent diffraction peaks must be measured to determine these 12 degrees of freedom, and ideally many more to reduce fitting error. The processing of the diffraction data had two major steps: indexing to associate diffraction peaks with a unique lattice orientation and data fitting to optimize the 12 grain parameters. The search space of orientations for the indexing procedure is generated from the measured diffraction peaks, noting that each can be used to define a crystallographic orientation fiber in the far-field geometry. The predicted locations of all diffraction peaks associated with a trial orientation are then tested locally in the measured data for intensity above a prescribed background level using a parallel algorithm [28]. Each trial orientation is scored by the ratio of detected versus expected reflections (referred to as completeness). Trial orientations having completeness scores above a prescribed threshold generally form dense clusters in the vicinity of the mutual intersection points of the source fibers in orientation space. Since the orientation of a grain will be the mutual intersection point of all orientation fibers associated with its observed diffraction peaks, these clusters are identified and associated with average orientations using a a density-based clustering algorithm applied over the orientation space [29].

After an approximate orientation is found during the indexing process, a forward modeling algorithm combined with a least squares minimization is used to find the optimized position, orientation, and strain parameters for each grain. In this work, overlapped diffraction peaks associated with a grain are discarded during the position, orientation, and strain minimization process. Typical uncertainties for grain position components, elastic strain components, and lattice orientation using experimental configurations similar to the one used in this work are approximately $1 / 10$ the detector pixel size $(20 \mu \mathrm{m}), 10^{-4}$, and $0.01^{\circ}$ respectively $[30,28,31]$.

\subsection{Determining Strengths from Grain Stress Data}

In this section, we describe the process of determining average slip system strengths from an ensemble of grain lattice strain tensors. Initially, the stress tensors of the grains are calculated from the lattice strain tensors using anisotropic linear elasticity. The elastic moduli used are listed in Table 1 [32]. From the full stress tensors, the resolved shear stresses on all basal $\langle a\rangle$, prismatic $\langle a\rangle$, pyramidal $\langle a\rangle$, and first-order pyramidal $\langle c+a\rangle$ slip systems are calculated. Next, the maximum resolved shear stress on each family of slip systems is found (e.g. the highest resolved shear stress on a basal $\langle a\rangle$ slip system in a grain). For a 
given grain, these are resolved shear stresses corresponding to slip systems that are likely to be dominant. Continuous probability distribution functions (PDFs) of the maximum resolved shear stresses from all grains in the ensemble are then generated using Gaussian kernel density estimation. To provide an example of these quantities, Figure 3 shows the distributions of per-family maximum resolved shear stress extracted from the experimental data at a macroscopic strain of 0.0154 . This load point is after plastic deformation has begun and is marked on Figure 1 with a red box. In Figure 3, normalized histograms of maximum resolved shear stresses of the four slip system families of interest are shown. The estimated continuous PDFs are plotted over the histograms with black dashed lines.

At this point, it is worth discussing how the slip system strengths are related to resolved shear stress PDFs such as those in Figure 3. To aid the description, Figure 4 provides idealized examples of different distributions. All PDFs described correspond to resolved shear stresses in ensembles of grains after the material has begun to plastically deform. A distribution of maximum resolved shear stresses in a rate-independent, perfectly plastic material is shown in Figure 4A. The distribution of resolved shear stresses on a set of slip systems during plastic deformation has a sharp cut-off at the slip system strength $\tau^{*}$ since these resolved shear stresses cannot exceed the strength anywhere in a grain. Note, that even in a rate-independent material, intra-granular stress heterogeneities will contribute to a broadening of the distributions of maximum resolved shear stresses across grain assemblies [33] and the far boundary of the distribution has a slight slope. Next, Figure 4B shows an example distribution from a set of grains that are no longer rate-independent. The grains will have a wider distribution of resolved shear stresses present because slip systems can be active under varying amounts of stress and there is no longer a sharp cut-off defining the slip system strength. Instead, we can define an average slip strength for the ensemble of grains that is halfway down the side of the distribution past the maximum value (the high stress side). In addition, we can define a width to this portion of the distribution $w_{\tau}$ that provides a measure of the spread of strengths across the ensemble of grains. Lastly, Figure 4C shows an example of the changes a resolved shear stress distribution undergoes with increasing strain if the grains are no longer perfectly plastic. Increases in grain slip system strengths due to hardening will have two effects on the distributions as deformation proceeds. First, the average slip system strengths $\tau^{*}$ will increase, pushing the high stress side of the distribution to the right. Second, the hardening will cause the width of the high 
stress side of the distribution to increase since all of the grains are not expected to harden at the same rate with respect to the increases in the macroscopic strain.

With the examples discussed, we return to the PDFs from the experimental data in Figure 3. To find both $\tau^{*}$ and $w_{\tau}$, analytic functions are fit to the high stress sides of the distributions. The form of the function $f$ is

$$
f=A\left(1-\tanh \left(\frac{2\left(\tau-\tau^{*}\right)}{w_{\tau}}\right)\right)
$$

where $A$ is a fitting constant. Examples of the fit functions are drawn with red dashed lines in Figure 3. As can be seen, the analytic function is sufficient to fit the high stress sides 230 of the PDFs. The process to find $\tau^{*}$ and $w_{\tau}$ was repeated for the slip system families of interest at all load steps beyond the elastic-plastic transition.

\section{Ti-7Al Modeling}

A crystal plasticity slip system strength model is introduced whose primary goal is to help understand, through modeling, the evolution of slip system strengths observed in the experiment and elucidate how the micromechanics influence the macroscopic response. In the model, the deformation gradient $\boldsymbol{F}$ is described with an elasto-viscoplastic decomposition into a plastic portion due to slip $\boldsymbol{F}_{P}$, a crystal lattice rotation $\boldsymbol{R}^{*}$, and a left elastic stretch tensor $\boldsymbol{V}_{E}[34,35]$

$$
\boldsymbol{F}=\boldsymbol{V}_{E} \cdot \boldsymbol{R}^{*} \cdot \boldsymbol{F}_{P}
$$

The plastic portion of the velocity gradient $\boldsymbol{L}_{P}$ is described as a linear combination of shearing motions on the active slip systems [36]

$$
\boldsymbol{L}_{P}=\dot{\boldsymbol{F}}_{P} \cdot \boldsymbol{F}_{P}^{-1}=\sum_{i} \dot{\gamma}^{(i)}\left(\boldsymbol{s}^{(i)} \otimes \boldsymbol{n}^{(i)}\right)
$$

where $i$ indicates a specific slip system, $\dot{\gamma}^{(i)}$ is a slip system shearing rate, $\boldsymbol{s}^{(i)}$ is a slip direction, and $\boldsymbol{n}^{(i)}$ is a slip plane normal in the appropriate configuration. The slip system shearing rates are governed by power law kinetics [37]

$$
\dot{\gamma}^{(i)}=\dot{\gamma}_{0}\left(\frac{\tau^{(i)}}{g^{(i)}}\right)\left|\frac{\tau^{(i)}}{g^{(i)}}\right|^{\frac{1}{m}-1}
$$

where $\dot{\gamma}_{0}$ is the reference shearing rate, $\tau^{(i)}$ is the resolved shear stress on the given slip

system, $m$ is the rate exponent, and $g^{(i)}$ is the resistance to slip of a slip system. 
The resistance to slip of a single slip system $g^{(i)}$ is given as

$$
g^{(i)}=g_{0}^{(i)}+q^{(i)} \sqrt{h_{\langle c+a\rangle}}+r \sqrt{\boldsymbol{M}: \boldsymbol{N}^{(i)}}
$$

where the first term $g_{0}^{(i)}$ is a constant slip resistance that varies for each family of slip systems.

The term $q^{(i)} \sqrt{h_{\langle c+a\rangle}}$ is a resistance to slip due to interactions of mobile dislocations on slip system $(i)$ with a forest of $\langle c+a\rangle$ dislocations. This interaction is a simplified form of general latent hardening models such as those that can be found in [38, 39]. The scalar $h_{\langle c+a\rangle}$ is the current dislocation density of $\langle c+a\rangle$ dislocations normalized by the initial dislocation density. The dislocation interaction terms $q^{(i)}$ consist of 4 independent parameters describing interactions between the $\langle c+a\rangle$ dislocation population and the families of slip systems modeled. Recent dislocation dynamics simulations of HCP metals have indicated that pyramidal $\langle c+a\rangle$ dislocations do not tend to serve as strong barriers to basal and prismatic slip [40]. We will therefore assume that interactions of dislocations associated with these slip systems with the $\langle c+a\rangle$ forest is negligible and the associated interaction parameters are equal to 0. Also, observations have been made that $\langle c+a\rangle$ dislocations combine to form immobile $\langle a\rangle$ dislocations [19] which we assume to be the primary contributor to the hardening of the pyramidal $\langle c+a\rangle$ slip systems. With these assumptions, only a single interaction term between the pyramidal $\langle c+a\rangle$ systems and the $\langle c+a\rangle$ forest remains.

The evolution of $h_{\langle c+a\rangle}$ is described with a Kocks-Mecking type relationship

$$
\dot{h}_{\langle c+a\rangle}=\left(k_{1} \sqrt{h_{\langle c+a\rangle}}-k_{2} h_{\langle c+a\rangle}\right) \sum_{i \in\langle c+a\rangle}\left|\dot{\gamma}^{(i)}\right|
$$

where $k_{1}$ and $k_{2}$ are model constants. Note that only slip on pyramidal $\langle c+a\rangle$ slip systems contributes to the evolution of $h_{\langle c+a\rangle}$.

The final term in Equation 5, which models the contribution to slip system strengths from the $\alpha_{2}$ precipitates, follows a less widely used form. The tensor $\boldsymbol{M}$ is an obstacle density tensor [41] used here to describe the distribution of $\alpha_{2}$ precipitates. The tensor $\boldsymbol{N}^{(i)}$ is a dyad constructed from the slip plane normal

$$
\boldsymbol{N}^{(i)}=\boldsymbol{n}^{(i)} \otimes \boldsymbol{n}^{(i)} .
$$

The term underneath the radical, $\boldsymbol{M}: \boldsymbol{N}^{(i)}$, is related to the spacing $L^{(i)}$ of $\alpha_{2}$ particles 
serving as obstacles to slip:

$$
L^{(i)}=\frac{1}{\sqrt{\boldsymbol{M}: \boldsymbol{N}^{(i)}}} .
$$

This mean free path is normalized by a characteristic length as to be unitless. The scalar $r$ is akin to a Taylor hardening coefficient for obstacles with a given characteristic spacing.

Generally, the initial obstacle density tensor $\boldsymbol{M}$ is symmetric and has six independent parameters. However, if we assume that initially the mean arrangement of $\alpha_{2}$ precipitates has the same symmetry as the hexagonal crystal lattice, only two independent coefficients remain [42]. In the crystal frame, only the diagonal terms are non-zero with $M_{11}$ being equal to $M_{22}$.

As slip occurs, the effective density of $\alpha_{2}$ precipitates drops as the particles are sheared due to dislocation motion [19]. To model this effect, the evolution of the obstacle density tensor $\boldsymbol{M}$ is described by an exponential decay

$$
\dot{\boldsymbol{M}}=-\eta \sum_{i}\left(\boldsymbol{M}: \boldsymbol{N}^{(i)}\right) \boldsymbol{N}^{(i)}\left|\dot{\gamma}^{(i)}\right|
$$

where $\eta$ is the precipitate destruction efficiency. The precipitate destruction efficiency controls how rapidly slip resistances will decrease with strain. As $\boldsymbol{M}$ decays, the mean free path between $\alpha_{2}$ precipitates increases, which in turn decreases the resistance to slip. At the point when $\boldsymbol{M}$ has decayed to $\mathbf{0}$, there is no longer a contribution to the slip system strength. We note that reduction of slip resistance has the tendency to promote increased localization of slip onto already active slip systems [43] consistent with observations of slip localization during titanium-aluminum alloy single crystal tests [15].

\subsection{Virtual Sample Generation}

Virtual specimens used to perform simulations were generated using the ff-HEDM data. The goal was to generate a microstructure that approximated the ensemble of grains probed during the experiment. First a $1 \mathrm{~mm} \times 2 \mathrm{~mm} \times 1 \mathrm{~mm}$ volume (along the $\boldsymbol{e}_{\boldsymbol{x}}^{S}, \boldsymbol{e}_{\boldsymbol{y}}^{S}$, and $\boldsymbol{e}_{\boldsymbol{z}}^{S}$ directions) was seeded with lattice orientations at specific points. In the center $1 \mathrm{~mm}$ of the volume along $\boldsymbol{e}_{\boldsymbol{y}}^{S}$, the lattice orientations measured from the experiment were seeded at grain centroids. At the top and bottom $500 \mu \mathrm{m}$ of the specimen, lattice orientations from the same orientation distribution as the experimentally tested specimen were seeded with randomly sampled points having the same point density as the center volume. The extra 
grains at the top and bottom of the virtual specimen serve as a buffer between the central $\langle c+a\rangle$ slip systems having high initial strengths.

The grain-by-grain stress data, in conjunction with the analysis just described, has allowed for the behavior of different families of slip systems to be analyzed independently. 
Equally important to note, the time resolution of the in-situ test makes it possible to observe softening of the basal $\langle a\rangle$, prismatic $\langle a\rangle$, and pyramidal $\langle a\rangle$ slip systems, and apparent hardening of the pyramidal $\langle c+a\rangle$ slip systems. These behaviors are not readily apparent in the macroscopic stress-strain response shown in Figure 1. In the macroscopic response, a slight softening followed by hardening is observed, but there is no way to partition this behavior on to the different families of slip systems. The magnitudes of these changes in the flow stress also are small enough that they can be overlooked. However, from the grain ensemble data, we find that the flat hardening response is actually caused by competing hardening and softening mechanisms in different sets of grains that combine to produce relatively little macroscopic hardening.

340 4.2. Calibrated Simulation Results

This section describes results using the slip system strength model described in the previous section. The model was implemented in the ALE3D finite element code developed at Lawrence Livermore National Laboratory [44]. More detailed descriptions of crystal plasticity implementations in ALE3D can be found in [35, 43]. All simulations were performed at a strain rate of $10^{-6} \mathrm{~s}^{-1}$, similar to that used during the experiment. Convergence of the macroscopic stress strain response with increasing numbers of elements is shown in Figure 7. It is expected that the flow stress level decreases slightly with mesh convergence. In finite element formulations of this sort [45], compatibility is enforced by construction and stress equilibrium is enforced in a weak sense. Therefore the results tend to be relatively stiff for coarser meshes. Reasonable convergence was found at 1024k total elements, and all slip system strength curves shown herein are from simulations with this many elements.

Plasticity parameters were found that reproduce the slip system strength evolution shown in Figure 6. The plasticity parameters associated with slip kinetics and dislocation interaction hardening are found in Table 2. In the experiment the sample was not deformed to large enough strains to observe any saturation of the strengths of the pyramidal $\langle c+a\rangle$ slip systems. Capturing saturation behavior is usually accomplished with the recovery term $k_{2} h_{\langle c+a\rangle}$ in the evolution equation of the dislocation density. Since, no saturation was observed, the recovery term is neglected for model calibration. The parameters associated with contributions of slip resistance due to the presence of $\alpha_{2}$ precipitates are listed in Table 3. The elastic moduli used in the simulations were the same as those used to process the diffraction data and are listed in Table 1. 
Figure 8 shows the slip system strength-strain curve from a simulation performed with $1024 \mathrm{k}$ elements. The calibrated model is able to reproduce the major features of the experimental slip system strength evolution. The average strengths of both the basal $\langle a\rangle$ and

components of the strength model and running the simulations. The three different cases that were tested were: removing hardening of the pyramidal $\langle c+a\rangle$ slip systems (labeled "no $\langle c+a\rangle$ hardening'), making the strength of the pyramidal $\langle c+a\rangle$ slip systems sufficiently 
high such that the activity of these slip systems is minimal (labeled 'strong $\langle c+a\rangle$ '), and removing the contribution to slip system strengths from the $\alpha_{2}$ precipitates (labeled 'no $\alpha_{2}$ strengthening'). The macroscopic stress strain curves from these simulations are found in Figure 9A.

The first case, no $\langle c+a\rangle$ hardening, has the same parameters as the calibrated model except that $k_{1}$ is equal to 0 . Setting $k_{1}$ to 0 removes all hardening from the pyramidal $\langle c+a\rangle$ slip systems. The slip system strength-strain curves for this simulation are shown in Figure 9B. Without the contribution to the slip system strengths from the hardening masking the contribution to the strength from the $\alpha_{2}$ precipitates, the $\langle c+a\rangle$ slip systems show significant softening that is not observed in the experiment. In addition, the macroscopic stress strain response has a much more pronounced decrease in the flow stress that is also not observed in the experiment. These simulations suggest that the hardening of the pyramidal $\langle c+a\rangle$ slip systems is the reason that softening due to obstacle shearing does not cause the same drop in strength as observed for the basal and prismatic slip systems.

The second case, strong $\langle c+a\rangle$, has the same parameters as the calibrated model except that the constant slip system resistances $g_{0}^{(i)}$ of the pyramidal $\langle c+a\rangle$ systems are raised by a factor of 3 to $1047 \mathrm{MPa}$. This increase in inital strength is consistent with existing assesments made from experimental observations [46, 15]. In this simulation, the grain stresses were not high enough to activate substantial amounts of pyramidal $\langle c+a\rangle$ slip. In the macroscopic response in Figure 9A, the only effect of the strengthening of the pyramidal $\langle c+a\rangle$ slip systems was to marginally increase the flow stress. However, if we look at the slip 415 system strength strain curves in Figure 9C, we can see that the average slip system strength $\tau^{*}$ of the $\langle c+a\rangle$ systems is much too high and the evolution of $\tau^{*}$ has significant curvature not seen in the experiment. Also, the reduced activity on the pyramidal $\langle c+a\rangle$ systems has a pronounced effect on the pyramidal $\langle a\rangle$ systems. The softening of the pyramidal $\langle a\rangle$ systems is much more distinct and the width of the distributions are decreased in comparison to the calibrated model. The fact that a simulation with strong $\langle c+a\rangle$ slip systems does not capture the evolution of $\tau^{*}$ is evidence that these slip systems are actually hardening as opposed to initiating with a higher strength without significant hardening.

The final case, no $\alpha_{2}$ strengthening, has the same parameters as the calibrated model except that the initial value of $\boldsymbol{M}$ is set to $\mathbf{0}$. The effect of removing the resistance to slip 425 due to the $\alpha_{2}$ precipitates in the macroscopic response is that the knee of the curve be- 
comes significantly less sharp. Also as would be expected, the average slip system strengths in Figure 9D show none of the softening that is observed in the experiment. Figure 5C shows the net slip at a macroscopic strain of 0.025 for this simulation. We can see in this simulation that the plastic deformation tends to localize in the same regions of crystal as the calibrated model. However, the localization of plastic deformation is less pronounced in the no $\alpha_{2}$ strengthening simulation indicating that the decrease in slip resistance caused by the shearing of the $\alpha_{2}$ precipitates tends to enhance the localization of slip in deforming grains. In total, these simulations show that both the dislocation-dislocation hardening and the decrease in the slip system strengths from the presence of $\alpha_{2}$ precipitates combine to produce the observed macroscopic response.

\section{Discussion}

The goals of this work were to demonstrate how ff-HEDM data can be used to identify and quantify slip system strength evolution during in-situ deformation and to show how these new data can be used to inform micromechanical modeling efforts. In this section, we discuss the implications of the results presented and provide suggestions for further avenues of research.

\subsection{Ti-7Al Response}

The macroscopic response of the sample exhibited a relatively flat work hardening response. Similar observations have been made concerning minimal work hardening in aged $445 \mathrm{Ti}-6 \mathrm{Al}$ and Ti-8Al polycrystalline specimens by Truax and McMahon Jr. [47] and aged Ti$7 \mathrm{Al}$ specimens by Fitzner et. al [17]. The low amounts of work hardening were attributed to an increase in slip localization that occurs in the presence of SRO and $\alpha_{2}$ precipitates. Our results confirm these conclusions: the decrease in strength attributed to shearing of $\alpha_{2}$ precipitates promoted enhanced slip localization in the simulations (Figure 5B and 5C). However, our results also indicate that hardening of the pyramidal $\langle c+a\rangle$ slip systems counteracts the slip localization on the basal and prismatic slip systems to prevent sharp drops in the flow stress at the elastic plastic transition. This observation is supported by the simulation in which pyramidal $\langle c+a\rangle$ hardening was removed and there was a pronounced decrease in the flow stress at yield (Figure 9A). All together, it appears that the transient 
455

dal $\langle c+a\rangle$ slip systems combined to produce the flat work hardening response observed in the tested $\mathrm{Ti}-7 \mathrm{Al}$. The presence of the dislocation obstacles also appears to contribute to the distinct yield point observed in the macroscopic response. When $\alpha_{2}$ strengthening was removed from the simulations, the yield point became much less distinct (Figure 9A).

The initial slip system strength values measured in this work for the prismatic and basal slip systems (248 and $253 \mathrm{MPa}$ respectively) are comparable to other measurements of critical resolved shear stress in elevated aluminum content single crystals. Williams et. al measured CRSS values of Ti-6.7Al single crystals in compression of 195 and $205 \mathrm{MPa}$ for the prismatic and basal slip systems [15], while May reported values of 279 and $293 \mathrm{MPa}$ for prismatic and basal slip systems for Ti-6.7Al single crystals deformed in tension [21]. In these works and in the present results, the presence of the aluminum content appears to lower the strength of the basal slip systems with respect to the prismatic slip systems. Also in agreement with observations made in this work, May observed a decrease in the flow stresses of the basal and prismatic slip systems at yield when the Ti-6.7Al single crystals were deformed in tension.

The experimental data showed that the $\langle c+a\rangle$ slip systems appeared to activate at much lower resolved shear stress levels $(\approx 270 \mathrm{MPa})$ than typically seen in the literature (785 [15] and $460 \mathrm{MPa}[21])$. However in the present experiment, the pyramidal $\langle c+a\rangle$ systems also appeared to rapidly harden and an average saturation value of the slip system strengths was never reached. These results suggest that the initial slip system strengths of pyramidal $\langle c+a\rangle$ slip systems in titanium alloys containing aluminum may show large amounts of variation. Depending on the amount of work hardening that has occurred, the pyramidal $\langle c+a\rangle$ slip systems may have relatively high slip system strengths, but this should not be automatically assumed to be true. Another possibility is that the rate sensitivity $m$ varies between the different families of slip systems. The experiment was performed at a very slow strain rate and if the rate sensitivity of the pyramidal $\langle c+a\rangle$ is higher than the other slip systems, the flow stress at higher rates will increase more rapidly than for other slip system types, consistent with other observations.

\subsection{Method Limitations and Future Work}

The values $\tau^{*}$ and $w_{\tau}$ are both measurable mesoscopic quantities that describe the distributions of stress in an ensemble of grains. There were two interpretations of these 
quantities that were driven by phenomenological arguments:

1. The stress value halfway down the high stress side of the PDF of maximum resolved shear stresses $\left(\tau^{*}\right)$ is the average slip system strength.

2. The width of the high stress side of the distribution $\left(w_{\tau}\right)$ is a measure of the spread of slip system strengths.

While the interpretation of these quantities can be debated, these values both appear to be intimately tied to the evolution of slip system strength in a deforming metal. Usually when trying to use macroscopic data to calculate slip system strengths, there are questions pertaining to the uniqueness of the microscale parameters that are used to produce macroscopic response. By capturing the evolution of quantities directly tied to the micromechancial response, we can be more confident that we are capturing the correct slip behavior at the grain scale.

During the calibration of the slip system strength model, the only data that were 'fit' were the slip system strength curves. As can be seen in Figure 1B, the slight inflections of the macroscopic stress-strain response, including the initial drop in the flow stress and then the subsequent increase, were naturally captured without having to make any changes to the model described in $\S 3$. In this respect, when fitting the slip system resistance from the mesoscale data, the bulk responses of the simulations are predictions that can be compared to the experimental results to judge how well the model is performing.

The ability to observe the short-transient softening behavior attributed to the destruction of $\alpha_{2}$ precipitates was possible because of the large number of load steps at which the sample was probed during the elastic-plastic transition. The need for large numbers of load steps to observe transient behavior highlights the need to continue to make improvements to data collection times. In the current work, the time limiting factor of the measurement process was detector collection time. With improvements to detector collection rates, studying numerous other transient behaviors such as the stick-slip behavior of flow, serrated yielding, and other dislocation breakaway mechanisms may be possible. Also, improvements in measurement time will allow for tests to be performed at higher strain rates and enable studies of slip kinetics.

In order to predict material failure, modeling efforts will need to be able to accurately capture the behavior of every grain in an ensemble in order to find weakest link grains. Before this point is reached however, there is still a great deal that can be learned from 
studying grains as ensembles via HEDM. As demonstrated in this work, one of the major utilities of the ff-HEDM method is the ability to probe the stress states of large numbers of grains simultaneously. With these data, we were able to gain new understanding of the aggregate response of the collections of grains and subsequently include that behavior in an improved constitutive model for this engineering alloy. These data show a great deal of promise for improving our ability to better predict behaviors such as texture evolution and 525 subsequently, mechanical properties on the macroscopic scale.

\section{Summary}

In this paper, ff-HEDM data is used to extract individual grain-average stress tensors in a large aggregate of Ti-7 Al grains $(\approx 500)$ over a time series of prescribed states during continuous tensile deformation in-situ. Distributions of the maximum resolved shear stresses over the ensemble of grains are calculated at each strain state, for each slip system family, to produce a corresponding slip system strength curve. These curves in turn provide the evolution of average strength for the associated slip system family. Namely, hardening of the pyramidal $\langle c+a\rangle$ slip systems, attributed to dislocation-dislocation interactions, and pronounced softening of the basal $\langle a\rangle$, prismatic $\langle a\rangle$, and pyramidal $\langle a\rangle$ slip systems, attributed to the destruction of ordered $\alpha_{2}$ precipitates, are identified. Using these data, a flow rule capable of replicating the slip system response is developed and calibrated. Lastly, crystal plasticity simulations are used to explore the effects of different portions of the slip system strength model and demonstrate how the relatively 'flat' macroscopic hardening response is caused by the competing hardening and softening mechanisms.

\section{Acknowledgments}

The authors would like to thank Jim Williams, Michael Mills, Armand Beaudoin, and TJ Turner for insightful discussions; Basil Blank, Ulrich Lienert, Ali Mashayekhi, and Jonathan Almer for help with the experiment; and Adam Pilchak for providing the Ti-7Al material. The work of Pagan, Barton, and Bernier was performed under the auspices of the U.S. Department of Energy by Lawrence Livermore National Laboratory under contract DEAC52-07NA27344 (LLNL-JRNL-703660). Support from the Materials and Manufacturing Directorate of the U.S. Air Force Research Laboratory is acknowledged. Use of the Advanced 
Photon Source, an Office of Science User Facility operated for the U.S. Department of Energy by Argonne National Laboratory, was supported under contract DEAC02-06CH11357.

[8] U. Lienert, M. Brandes, J. Bernier, J. Weiss, S. Shastri, M. Mills, M. Miller, In situ single-grain peak profile measurements on Ti7Al during tensile deformation, Materials Science and Engineering: A 524 (2009) $46-54$.

[9] L. Wang, J. Lind, H. Phukan, P. Kenesei, J.-S. Park, R. Suter, A. Beaudoin, T. Bieler, 575

[1] J. Allison, D. Backman, L. Christodoulou, Integrated computational materials engineering: A new paradigm for the global materials profession, JOM 58 (2006) 25-27.

[2] J. H. Panchal, S. R. Kalidindi, D. L. McDowell, Key computational modeling issues in integrated computational materials engineering, Computer-Aided Design 45 (2013) 4

[3] A. A. Benzerga, J.-B. Leblond, Ductile fracture by void growth to coalescence, in: H. Aref, E. van der Giessen (Eds.), Advances in Applied Mechanics, volume 44 of Advances in Applied Mechanics, Elsevier, 2010, pp. 169 - 305.

[4] R. A. Lebensohn, J. P. Escobedo, E. K. Cerreta, D. Dennis-Koller, C. A. Bronkhorst, J. F. Bingert, Modeling void growth in polycrystalline materials, Acta Materialia 61 (2013) $6918-6932$.

[5] H. Poulsen, Three-Dimension X-Ray Diffraction Microscopy, 1st ed., Springer, 2004.

[6] M. Miller, P. Dawson, Understanding local deformation in metallic polycrystals using high energy x-rays and finite elements, Current Opinion in Solid State and Materials Science 18 (2014) $286-299$.

[7] J. C. Schuren, P. A. Shade, J. V. Bernier, S. F. Li, B. Blank, J. Lind, P. Kenesei, U. Lienert, R. M. Suter, T. J. Turner, D. M. Dimiduk, J. Almer, New opportunities for quantitative tracking of polycrystal responses in three dimensions, Current Opinion in Solid State and Materials Science 19 (2015) 235 - 244. Opportunities in Mesoscale Science.

Mechanical twinning and detwinning in pure Ti during loading and unloading an in 
situ high-energy x-ray diffraction microscopy study, Scripta Materialia 92 (2014) 35 38.

[10] K. Chatterjee, A. Venkataraman, T. Garbaciak, J. Rotella, M. Sangid, A. Beaudoin, P. Kenesei, J.-S. Park, A. Pilchak, Study of grain-level deformation and residual stresses in ti-7al under combined bending and tension using high energy diffraction microscopy (hedm), International Journal of Solids and Structures 9495 (2016) 35 - 49.

[11] T. J. Turner, P. A. Shade, J. V. Bernier, S. F. Li, J. C. Schuren, P. Kenesei, R. M. Suter, J. Almer, Crystal plasticity model validation using combined high-energy diffraction microscopy data for a Ti-7Al specimen (In Press).

[12] G. Lutjering, J. Williams, Titanium, 2nd ed., Springer-Verlag, 2007.

[13] J. C. Williams, M. J. Blackburn, The identification of a non-basal slip vector in titanium and titanium-aluminum alloys, physica status solidi (b) 25 (1968) K1-K3.

[14] T. Cass, Slip modes and dislocation substructures in titanium and titanium-aluminum single crystals, in: R. Jaffee, N. Promisel (Eds.), The Science, Technology and Application of Titanium, 1970, pp. 733-739.

[15] J. C. Williams, R. G. Baggerly, N. E. Paton, Deformation behavior of hcp Ti-Al alloy single crystals, Metallurgical and Materials Transactions A 33 (2002) 837-850.

[16] A. Venkataraman, P. A. Shade, S. S. R. Adebisi, A. L. Pilchak, G. B. Viswanathan, M. C. Brandes, M. J. Mills, M. D. Sangid, Study of structure and deformation pathways in Ti-7 Al using atomistic simulations, experiments and characterization (Under Review).

[17] A. Fitzner, D. L. Prakash, J. Q. da Fonseca, M. Thomas, S.-Y. Zhang, J. Kelleher, P. Manuel, M. Preuss, The effect of aluminium on twinning in binary alpha-titanium, Acta Materialia 103 (2016) 341 - 351.

[18] T. Neeraj, M. Mills, Short-range order (SRO) and its effect on the primary creep behavior of a Ti6wt.\%Al alloy, Materials Science and Engineering: A 319321 (2001) $415-419$. 
[19] M. J. Blackburn, J. C. Williams, Strength, deformation modes and fracture in titaniumaluminum alloys, ASM Quarterly Transactions 62 (1969) 398-407.

[20] M. Brandes, M. J. Mills, Static recovery in titanium alloys at lower temperatures, Materials Science and Engineering: A 387389 (2004) 570 - 575. 13th International Conference on the Strength of Materials.

[21] K. May, Small Scale Tensile Testing of Titanium Alloys, Master's thesis, The Ohio State University, 2010.

[22] A. L. Pilchak, Fatigue crack growth rates in alpha titanium: Faceted vs. striation growth, Scripta Materialia 68 (2013) $277-280$.

[23] P. A. Shade, B. Blank, J. C. Schuren, T. J. Turner, P. Kenesei, K. Goetze, R. M. Suter, J. V. Bernier, S. F. Li, J. Lind, U. Lienert, J. Almer, A rotational and axial motion system load frame insert for in situ high energy x-ray studies, Review of Scientific Instruments 86 (2015) 093902.

[24] M. Guizar-Sicairos, S. T. Thurman, J. R. Fienup, Efficient subpixel image registration algorithms, Opt. Lett. 33 (2008) 156-158.

[25] P. A. Shade, D. B. Menasche, J. V. Bernier, P. Kenesei, J.-S. Park, R. M. Suter, J. C. Schuren, T. J. Turner, Fiducial marker application method for position alignment of in situ multimodal X-ray experiments and reconstructions, Journal of Applied Crystallography 49 (2016) 700-704.

[26] M. Obstalecki, S.-L. Wong, P. Dawson, M. Miller, Quantitative analysis of crystal scale deformation heterogeneity during cyclic plasticity using high-energy x-ray diffraction and finite-element simulation, Acta Materialia 75 (2014) 259 - 272.

[27] J. H. Lee, C. C. Aydiner, J. Almer, J. Bernier, K. W. Chapman, P. J. Chupas, D. Haeffner, K. Kump, P. L. Lee, U. Lienert, A. Miceli, G. Vera, Synchrotron applications of an amorphous silicon flat-panel detector, Journal of Synchrotron Radiation 15 (2008) $477-488$.

[28] J. Bernier, N. Barton, U. Lienert, M. Miller, Far-field high-energy diffraction microscopy: a tool for intergranular orientation and strain analysis, The Journal of Strain Analysis for Engineering Design 46 (2011) 527-547. 
[29] M. Ester, H. Kriegel, J. Sander, X. Xu, A density-based algorithm for discovering clusters in large spatial databases with noise, in: Proceedings of the 2nd International Conference on Knowledge Discovery and Data Mining, 1996, pp. 226-231.

[30] J. Oddershede, S. Schmidt, H. Poulsen, H. O. Sørensen, J. Wright, W. Reimers, Determining grain resolved stresses in polycrystalline materials using three-dimensional X-ray diffraction, Journal of Applied Crystallography 43 (2010) 539-549.

[31] H. Sharma, R. M. Huizenga, S. E. Offerman, A fast methodology to determine the characteristics of thousands of grains using three-dimensional X-ray diffraction. II. Volume, centre-of-mass position, crystallographic orientation and strain state of grains, Journal of Applied Crystallography 45 (2012) 705-718.

[32] E. S. Fisher, C. J. Renken, Single-crystal elastic moduli and the hcp $\rightarrow$ bcc transformation in ti, zr, and hf, Phys. Rev. 135 (1964) A482-A494.

[33] U. Lienert, S. F. Li, C. M. Hefferan, J. Lind, R. M. Suter, J. V. Bernier, N. R. Barton, M. C. Brandes, M. J. Mills, M. P. Miller, B. Jakobsen, W. Pantleon, High-energy diffraction microscopy at the advanced photon source, JOM 63 (2011) 70-77.

[34] E. Marin, P. Dawson, On modelling the elasto-viscoplastic response of metals using polycrystal plasticity, Computer Methods in Applied Mechanics and Engineering 165 (1998) $1-21$.

[35] N. R. Barton, N. W. Winter, J. E. Reaugh, Defect evolution and pore collapse in crystalline energetic materials, Modelling and Simulation in Materials Science and Engineering 17 (2009) 035003.

[36] R. Asaro, Crystal plasticity, Journal of Applied Mechanics 50 (1983) 921-934.

[37] H. Mecking, U. Kocks, Kinetics of flow and strain-hardening, Acta Metallurgica 29 (1981) $1865-1875$.

[38] P. Franciosi, M. Berveiller, A. Zaoui, Latent hardening in copper and aluminium single crystals, Acta Metallurgica 28 (1980) $273-283$.

[39] F. Lavrentev, Y. Pokhil, Relation of dislocation density in different slip systems to work hardening parameters for magnesium crystals, Materials Science and Engineering $18(1975) 261-270$. 
[40] M. Messner, M. Rhee, A. Arsenlis, N. Barton, A crystal plasticity model for slip resistance and junction formation in hcp metals (Submitted).

[41] T. P. Harrigan, R. W. Mann, Characterization of microstructural anisotropy in orthotropic materials using a second rank tensor, Journal of Materials Science 19 (1984)

[44] B. Stiller, T. Bocek, F. Hecht, G. Machado, P. Racz, M. Waldburger, Users manual for ALE3D - an arbitrary Lagrange/Eulerian 3D code system. Technical Report UCRLMA-152204, Technical Report, Lawrence Livermore National Laboratory, 2003.

[45] F. Roters, P. Eisenlohr, L. Hantcherli, D. Tjahjanto, T. Bieler, D. Raabe, Overview

[47] D. Truax, C. M. Jr., Plastic behavior of titanium-aluminum alloys, Materials Science and Engineering 13 (1974) 125 - 139. 


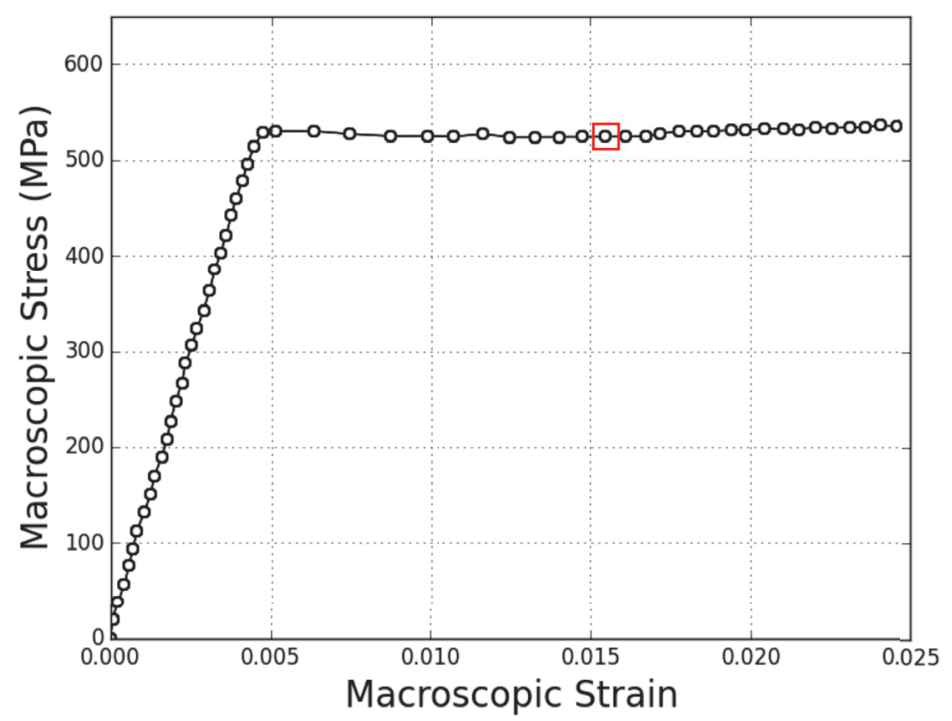

Figure 1: A) Macroscopic stress strain data from the tension experiment. A red box is drawn around the load point at which experimental distribution data are shown in Figure 3. 


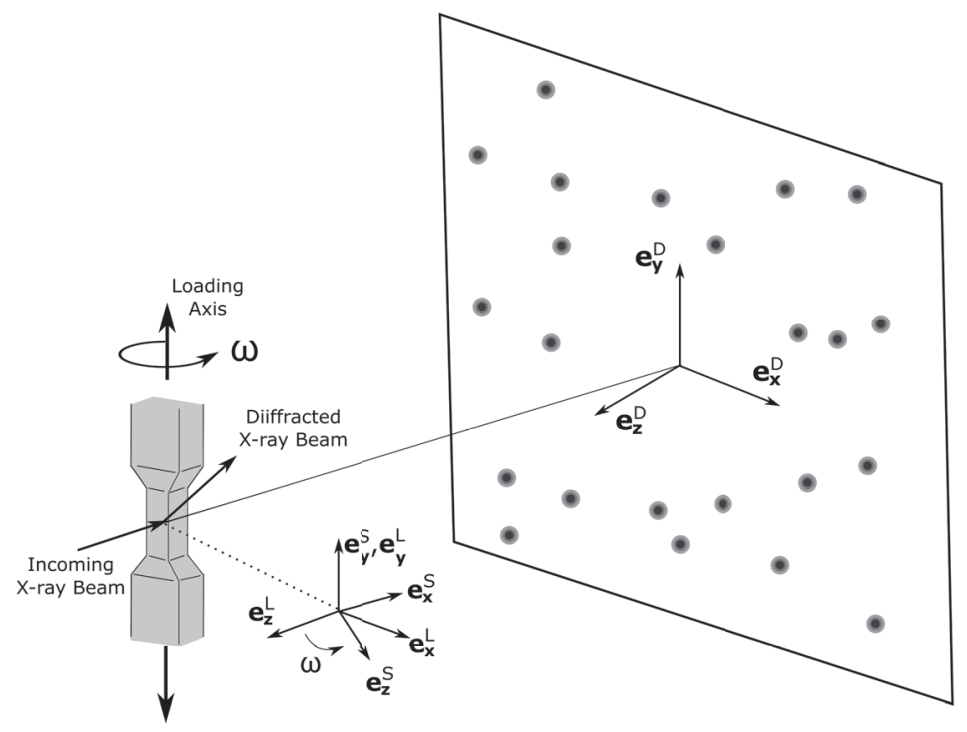

Figure 2: Schematic of the experimental geometry of the ff-HEDM experiment. The laboratory, sample, and detector coordinate systems are labeled with $\mathrm{L}, \mathrm{S}$, and $\mathrm{D}$ respectively. The incoming beam traveled in the $-\boldsymbol{e}_{\boldsymbol{z}}^{L}$ direction. The sample was loaded and rotated about $\boldsymbol{e}_{\boldsymbol{y}}^{L}$ by the angle $\omega$. When $\omega=0^{\circ}$, the laboratory and sample coordinate systems are in coincidence. 

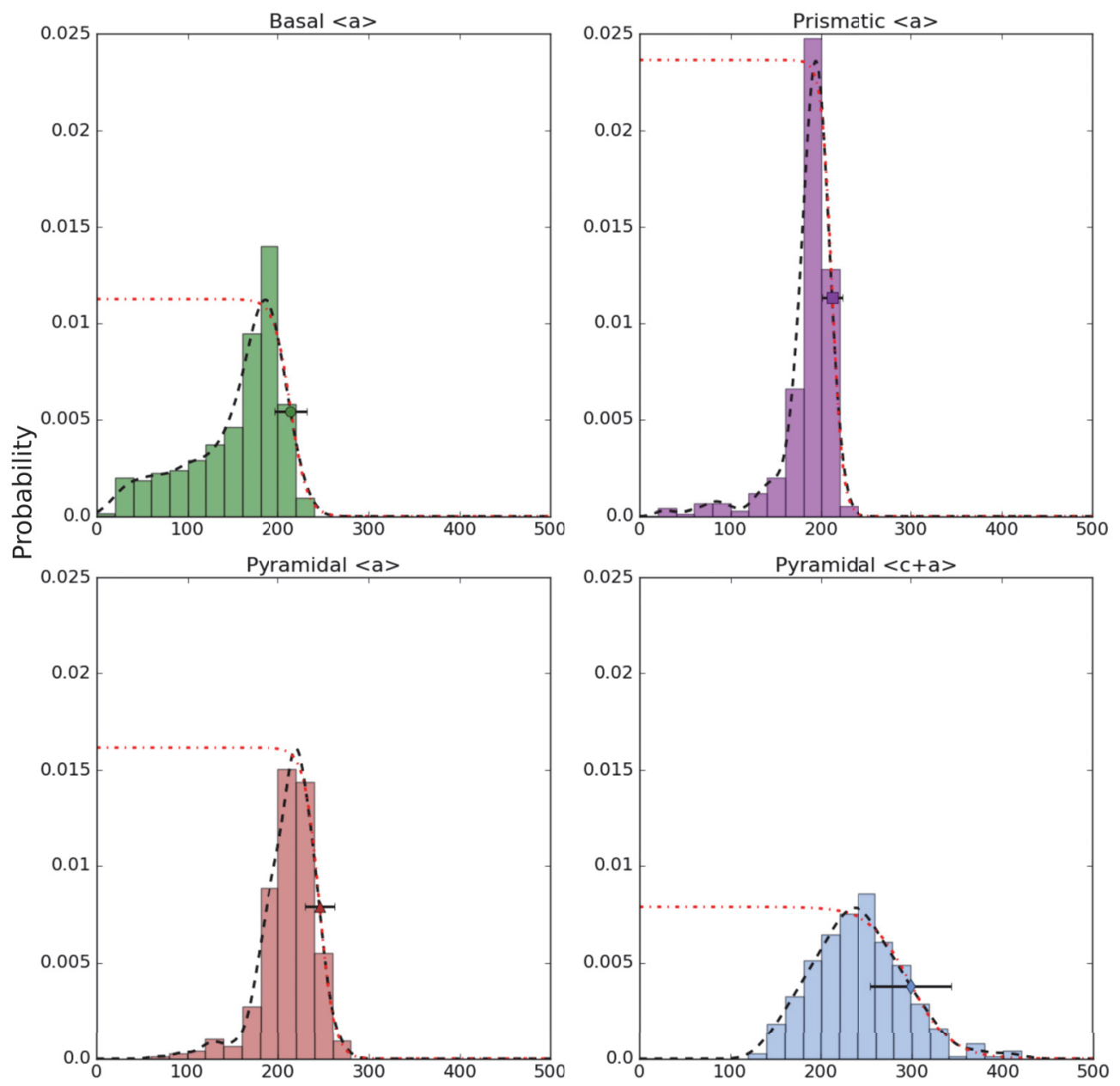

Maximum Resolved Shear Stresses (MPa) for Each Slip System Family, Macroscopic Strain $=0.0154$

Figure 3: Normalized histograms of the per-family maximum resolved shear stresses of the grain ensemble at a macroscopic strain of 0.0154 . Estimated continuous probability distribution functions are drawn with dashed black lines. The analytic functions fit to the high stress side of the probability distributions are drawn with dashed red lines. The estimated average slip systems $\tau^{*}$ are marked with a glyph and the spreads of the distributions $w_{\tau}$ are drawn with errorbars. 


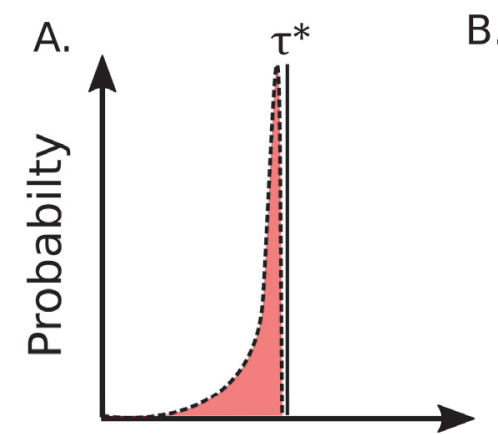

B.

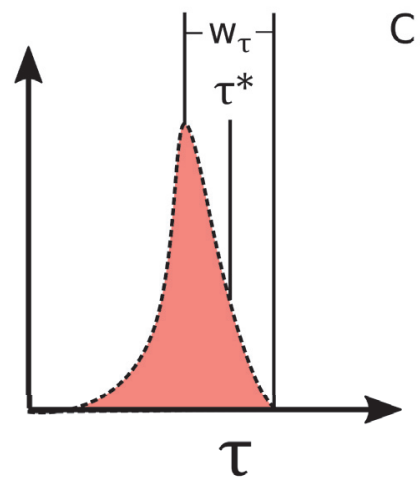

C.

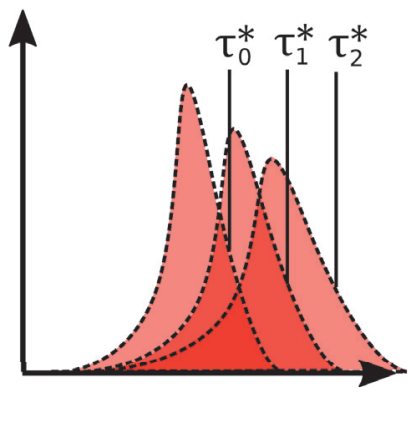

Figure 4: A) An example probability distribution function (PDF) of maximum resolved shear stresses applied to a family of slip systems in an ensemble of rate-independent, perfectly plastic grains. B) An example PDF of maximum resolved shear stresses applied to a slip system family in an ensemble of grains that are ratedependent, but perfectly plastic. The average slip system strength is $\tau^{*}$ and the spread of the distribution is $w_{\tau}$. C) The effects of slip system hardening on the evolution of PDFs of maximum resolved shear stresses applied to a slip system family for an ensemble of grains. 
A)

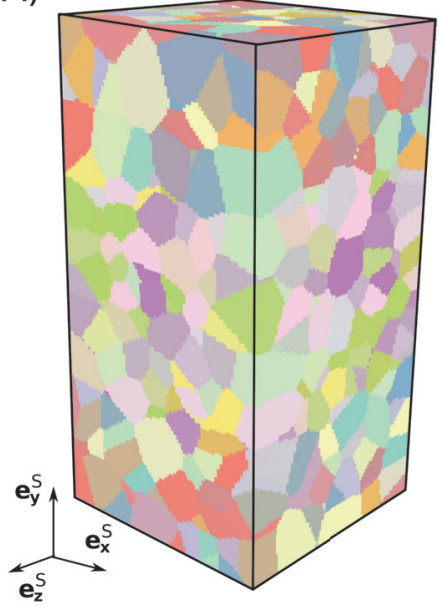

B)

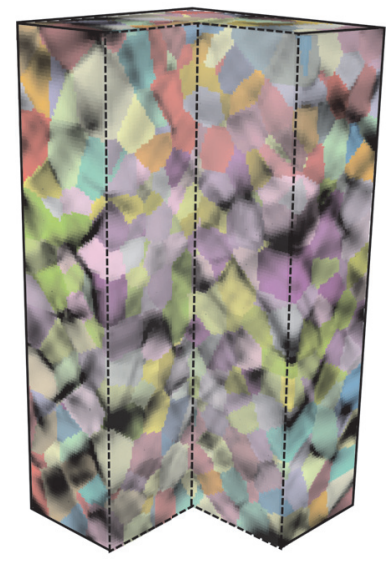

C)

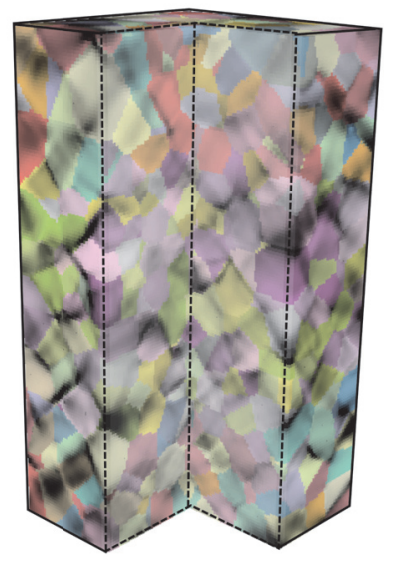

Figure 5: A) Microstructure generated using grain centroids and initial orientations determined from ffHEDM data. B) The net slip $\left(\sum\left|\dot{\gamma}^{(i)}\right|\right)$ overlaid over the grain microstructure at a macroscopic strain of 0.025 calculated from the calibrated model. C) The net slip overlaid over the grain microstructure at a macroscopic strain of 0.025 calculated from the model while neglecting the contribution to slip system strength from the $\alpha_{2}$ precipitates. Black corresponds to a net slip greater than $0.1 \mathrm{~s}^{-1}$. 

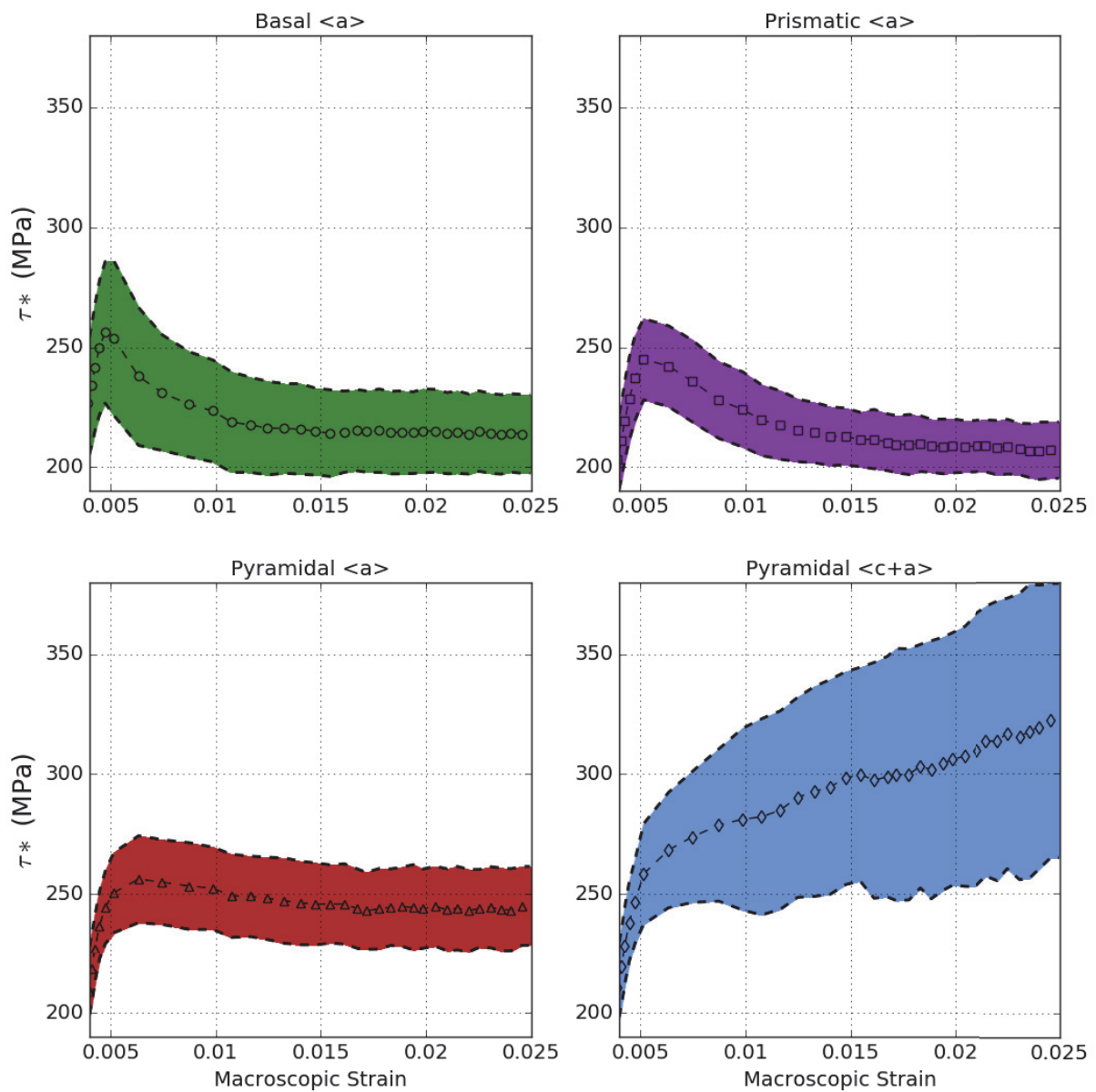

Figure 6: Slip system strength strain curves for the four slip system families of interest calculated from the experimental data. The glyphs mark the average slip system strengths $\tau^{*}$ across the collection of grains probed and the colored regions indicated the spread of the slip system strengths $w_{\tau}$. 


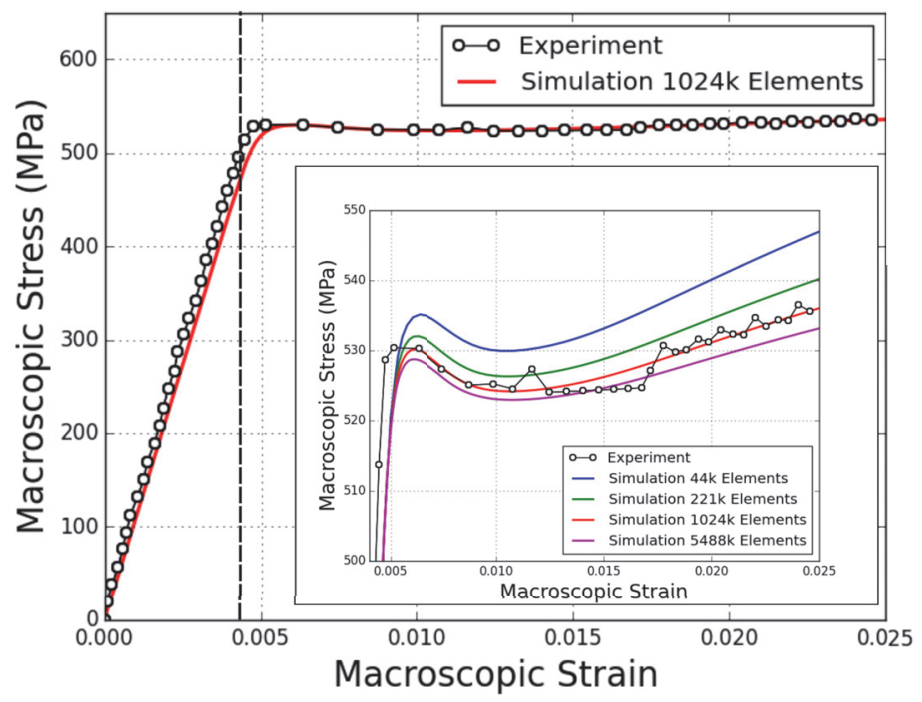

Figure 7: Macroscopic stress strain data from the tension experiment and the calibrated model with $1024 \mathrm{k}$ elements. Inset: Detailed macroscopic stress responses past the elastic-plastic transition with $44 \mathrm{k}, 221 \mathrm{k}$, $1024 \mathrm{k}$, and $5488 \mathrm{k}$ elements. 

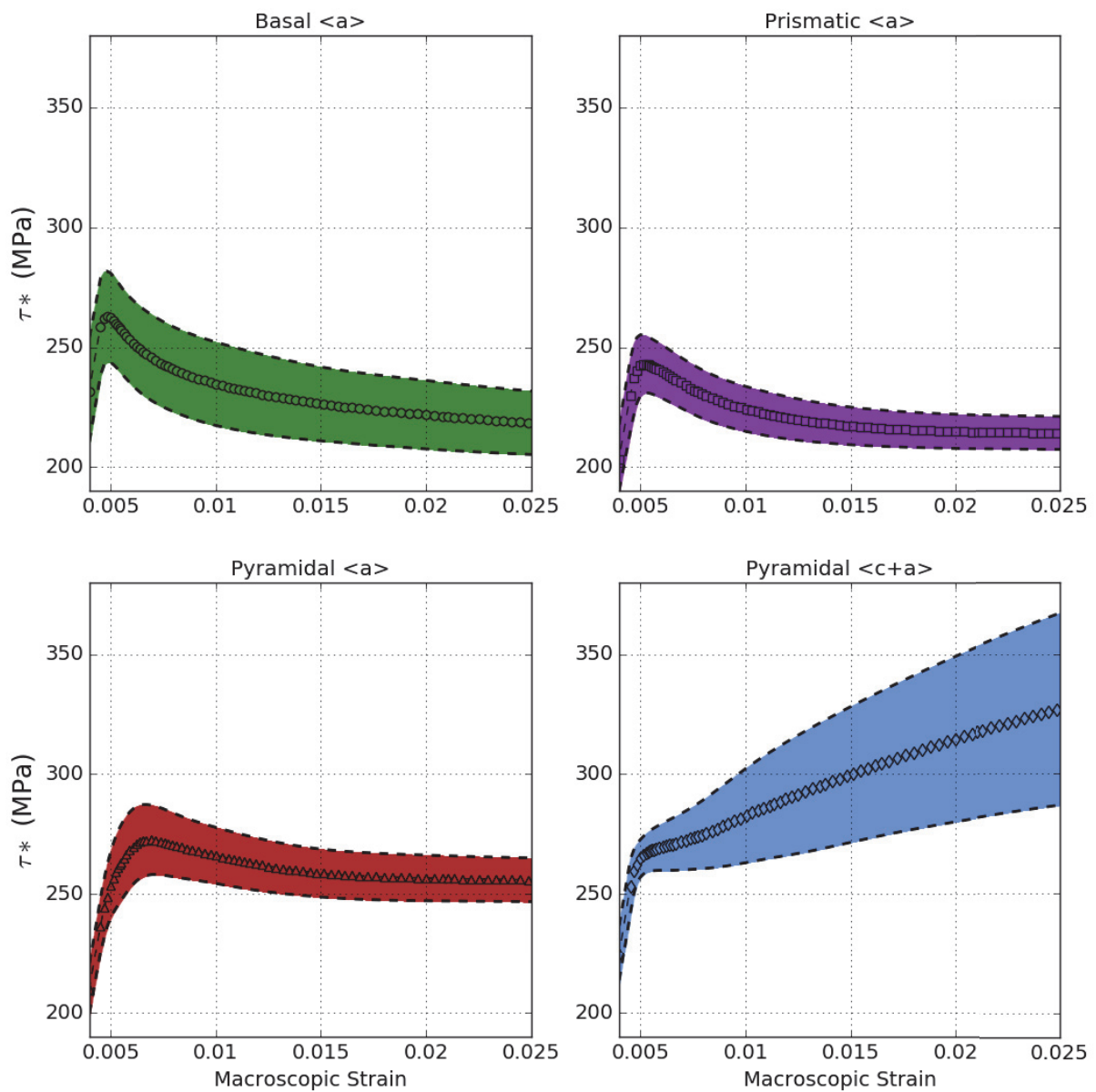

Figure 8: Slip system strength strain curves for the four slip system families of interest calculated from the calibrated model with $1024 \mathrm{k}$ elements. 
A)

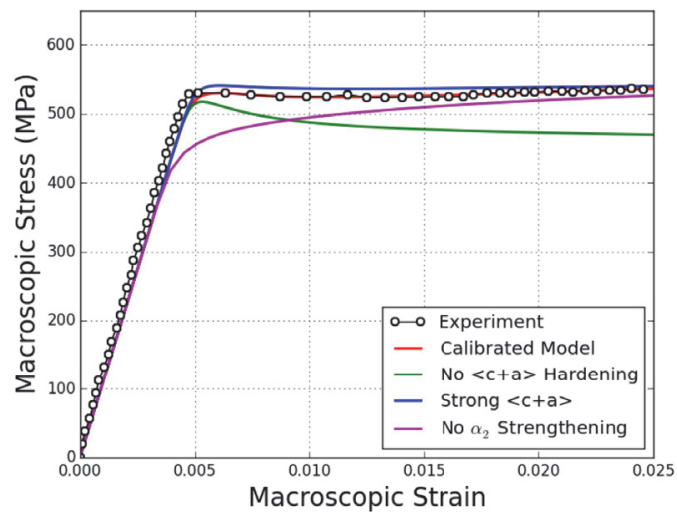

C)

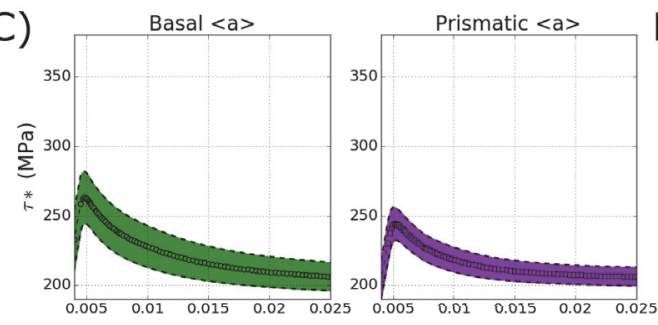
Pyramidal $<a>$

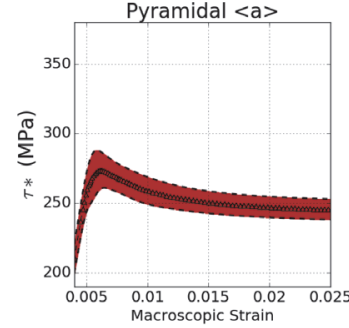

B)
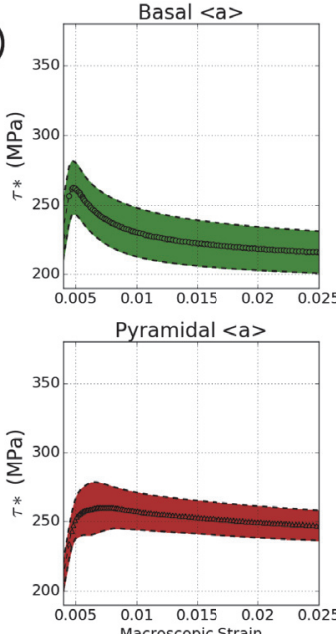

0.005
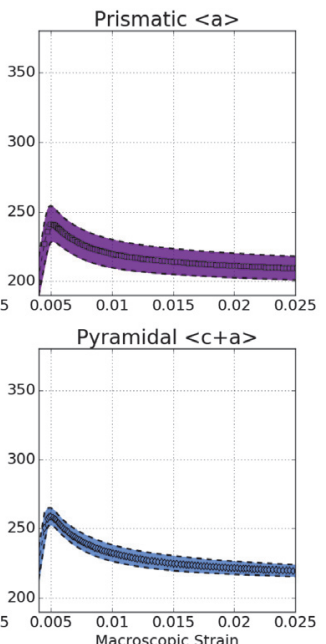

Basal $<a>$
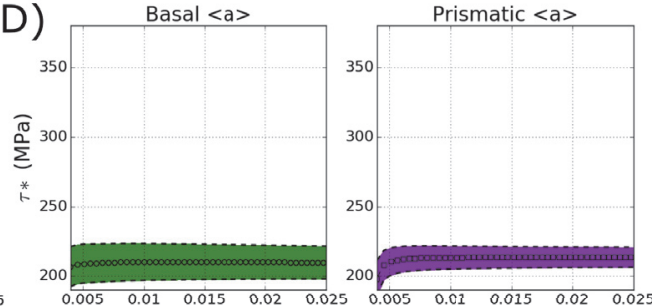

Pyramidal $<a>$

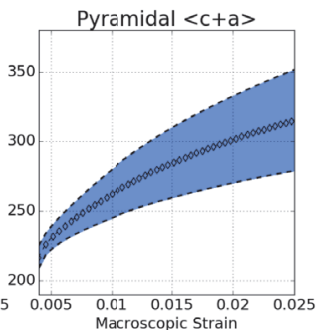

Figure 9: A) Macroscopic stress strain curves from four different simulations. The simulations correspond to the calibrated model, a simulation with no hardening of the pyramidal $\langle c+a\rangle$ slip systems, a simulation with high initial slip slip system strengths of the pyramidal $\langle c+a\rangle$ slip systems, and a simulation with no contribution to the slip system strengths from $\alpha_{2}$ precipitates. B) Slip system strength strain curves from the simulations with no hardening of the pyramidal $\langle c+a\rangle$ slip systems. C) Slip system strength strain curves from the simulations with high initial slip system strengths of the pyramidal $\langle c+a\rangle$ slip systems. D) Slip system strength strain curves from the simulations with no contribution to slip resistance from $\alpha_{2}$ precipitates. 


\begin{tabular}{c|c|c|c|c}
$C_{11}$ & $C_{12}$ & $C_{13}$ & $C_{33}$ & $C_{44}$ \\
\hline 162.4 & 92.0 & 69.0 & 180.7 & 46.7
\end{tabular}

Table 1: Elastic stiffness constants in GPa used in this work [32]. 


\begin{tabular}{c|c|c|c|c|c|c|c|c|c|c|c}
\multicolumn{1}{c|}{} & \multicolumn{3}{c|}{ Basal $\langle a\rangle$} & \multicolumn{2}{|c|}{ Prism. $\langle a\rangle$} & \multicolumn{2}{c|}{ Pyr. $\langle a\rangle$} & \multicolumn{2}{|c|}{ Pyr. $\langle c+a\rangle$} \\
\hline$m$ & $\dot{\gamma}_{0}$ & $k_{1}$ & $k_{2}$ & $g_{0}$ & $q$ & $g_{0}$ & $q$ & $g_{0}$ & $q$ & $g_{0}$ & $q$ \\
\hline 0.02 & $10^{6}$ & 1700 & 0 & 328 & 0 & 342 & 0 & 417 & 0 & 349 & 20
\end{tabular}

Table 2: Calibrated plasticity model parameters associated with slip kinetics and slip system hardening. Values of $g_{0}$ and $q$ have units of MPa and $\dot{\gamma}_{0}$ has units of $1 / \mathrm{s}$. All other parameters are unitless. 


\begin{tabular}{c|c|c|c|c|c|c|c}
$\eta$ & $r$ & $M_{11}$ & $M_{22}$ & $M_{33}$ & $M_{23}$ & $M_{13}$ & $M_{12}$ \\
\hline 365 & 11 & 35 & 35 & 90 & 0 & 0 & 0
\end{tabular}

Table 3: Calibrated plasticity model parameters associated with slip resistance due to the presence of $\alpha_{2}$ precipitates. The parameter $r$ has units of $\mathrm{MPa}$. All other parameters are unitless. Values of $\boldsymbol{M}$ are given in the crystal coordinate system. 